\section{Cahiers balkaniques}

$36-37 \mid 2008$

L'image de la période ottomane dans les littératures balkaniques

\title{
L'image du Turc dans la poésie épique serbe
}

The image of Turk in the epic serbian poetry

\section{Sanja Bošković}

\section{CpenEdition}

\section{Journals}

Édition électronique

URL : https://journals.openedition.org/ceb/1490

DOI : $10.4000 /$ ceb. 1490

ISSN : 2261-4184

Éditeur

INALCO

\section{Édition imprimée}

Date de publication : 1 décembre 2008

Pagination : 79-98

ISBN : 978-2-85831-173-6

ISSN : 0290-7402

\section{Référence électronique}

Sanja Bošković, "L'image du Turc dans la poésie épique serbe », Cahiers balkaniques [En ligne], 36-37 2008, mis en ligne le 15 mai 2012, consulté le 06 juillet 2021. URL : http://journals.openedition.org/ ceb/1490 ; DOI : https://doi.org/10.4000/ceb.1490

Ce document a été généré automatiquement le 6 juillet 2021.

\section{c) (i) (8)}

Cahiers balkaniques est mis à disposition selon les termes de la Licence Creative Commons Attribution - Pas d'Utilisation Commerciale 4.0 International. 


\section{L’image du Turc dans la poésie épique serbe}

The image of Turk in the epic serbian poetry

\section{Sanja Bošković}

L'un des plus grands mérites de Vuk Stéfanovitch - Karadjitch (1787-1864), le réformateur du serbe moderne et l'auteur du premier dictionnaire publié en 1818, est son travail de collecteur ardent de l'art populaire. Persuadé que la poésie épique serbe, fruit de la riche tradition orale, porte une valeur exceptionnelle dans la formation de la langue elle-même ainsi que dans la constitution de l'identité nationale et de la mémoire collective, Karadjitch se hâte, durant toute sa vie, de rencontrer les chanteurs épiquesaèdes pour les écouter et noter les poèmes. C'est ainsi qu'il réussit à recueillir environ mille chants populaires. En les publiant en 1823, à Vienne, dans un recueil en quatre volumes, il les sauve de l'oubli et leur donne une nouvelle vie. À l'époque où Karadjitch agit et édite la plupart de ses œuvres, la capitale autrichienne joue le rôle du foyer intellectuel des Slaves du Sud où se croisent non seulement les aspirations culturelles et politiques venues des Balkans, mais aussi celles des savants de l'Europe centrale du XIX siècle. Là Karadjitch rencontre son futur ami et collègue Jernej Bartholomé Kopitar, grammairien et slavisant réputé, grâce à qui son travail de collecteur a pu être communiqué à un public de mérite ${ }^{1}$.

Pour les romantiques comme Goethe et Grimm, la poésie épique serbe représente une véritable découverte. L'auteur du Jeune Werther montrera un intérêt particulier: il traduit en allemand le poème La triste chanson de la noble femme d'Assan Aga et publie plusieurs chants dans sa revue littéraire. Grâce à son amitié avec Grimm, Claude Fauriel devient, de son côté, en France, l'un des premiers connaisseurs de la tradition populaire serbe. Portant un regard comparatif sur les différentes traditions folkloriques, il consacre, l'hiver 1831-1832, aux poésies grecque et serbe, onze leçons de son enseignement à la Sorbonne ${ }^{2}$.

Les critères de systématisation de Vuk Karadjitch prennent en compte le contenu thématique des poèmes. Dans son recueil, il divise les chants en deux familles: les poèmes féminins traitant des sujets appropriés, c'est-à-dire les mariages, les travaux et 
les rites domestiques, l'amour, etc... et les poèmes masculins qui évoquent les thèmes héroïques, les batailles, les guerres, les personnages et les événements importants de l'histoire.

Les chants masculins sont au centre de nos recherches dans cette étude. Ces poèmes qui parlent des batailles sanglantes et de l'héroïsme national se situent historiquement à l'époque de l'envahissement turc des territoires serbes. Selon les différentes périodes de l'occupation ottomane, on peut diviser cette catégorie de chants en quatre cycles : le pivot du premier est la célèbre bataille de Kossovo qui a eu lieu le 27 juin 1389 sur le Champ des Merles; vient ensuite l'époque de vassalité où le héros emblématique Marko Kralievitch (le prince Marko), tout en acceptant la domination turque, essaye de défendre l'identité nationale et religieuse de ses compatriotes. Le troisième cycle contient les poèmes sur les haïdouks, les brigands des grandes routes, qui, vivant dans les forêts et menant le combat individuel contre les Turcs, se montrent comme les prédécesseurs des guerriers de libération qui à partir de 1804 s'engagent, de manière organisée, à pourchasser l'envahisseur. Le dernier cycle des chants héroïques célèbre donc la guerre de l'Indépendance et le retour de la liberté.

\section{Le cycle de Kossovo}

L'arrivée des Turcs, le choc culturel et religieux qu'elle provoque marque profondément l'esprit du peuple serbe. Le désarroi dû à la perte de l'indépendance et l'espoir du retour de la liberté sont les deux préoccupations qui dominent les chants héroïques. En même temps, l'épopée serbe esquisse le portrait de l'envahisseur turc qui, avec la force d'un destin tragique, frappe et imprègne l'imaginaire.

Les poèmes du cycle de Kossovo illustrent le drame d'un peuple anéanti par l'arrivée brusque d'une religion et d'une civilisation nouvelles, au point que, après la défaite militaire en 1389, son identité est mortellement menacée. Bien que le cycle des chants consacré à la bataille de Champs des Merles ne soit pas entièrement conservé - nous ne disposons que de quelques longs fragments -, il est possible d'entrevoir l'affrontement terrible des deux armées.

Dans la partie conservée du poème La bataille de Kossovo, le chanteur décrit l'afflux des soldats turcs sur le champ de combat et l'envoi de la lettre du sultan Murad au tzar serbe Lazar dans laquelle le souverain turc exige :

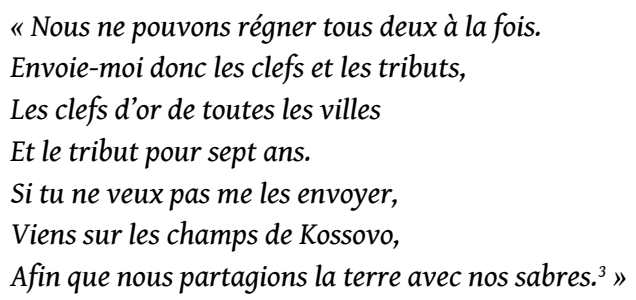

Ne pouvant pas accepter l'ultimatum du sultan Murad, le tzar Lazar, avec tous les autres princes serbes, décide de se rendre au Kossovo et de livrer le combat malgré la supériorité en nombre et en force de l'ennemi. C'est la raison pour laquelle, Lazar lance un appel à tout son royaume en maudissant les traîtres :

«Maudit soit celui qui ne vient pas au Kossovo!

Que de sa main rien ne naisse,

Ni le blé blanc dans les champs,

Ni les belles vignes dans les monts !" " 
L'importance de l'armée turque est confirmée par un autre fragment préservé où le héros Kosantchitch Ivan informe sur l'état de l'armée turque le célèbre Miloche obilitch, le guerrier hérö̈que qui tuera le sultan Murad pendant la bataille de Kossovo :

"O mon frère, Miloche Obilitch,

Oui, j'ai reconnu l'armée turque :

C'est une troupe immense.

Fussions-nous tous, nous Serbes, changés en sel,

Nous ne salerions pas la nourriture des Turcs.

Voilà deux semaines entières

Que je me dirige chaque jour vers les hordes turques,

Et je n'ai pu en trouver ni la fin, ni le nombre. ${ }^{5}$ "

L'importance de l'armée turque bien plus nombreuse que l'armée serbe est présentée dans les vers comme une force puissante et expérimentée. Le poète souligne cette puissance en décrivant les préparatifs soignés des soldats serbes. Ils sont des milliers et des milliers à arriver au Kossovo, prêts à combattre les Turcs et «verser le sang pour la Sainte Croix et la liberté d'or ${ }^{6} »$. Pourtant, tous leurs efforts et leur résolution de se sacrifier pour la défense de leur empire restent insuffisants devant un ennemi aussi vigoureux. Partir à la guerre, pour les Serbes, est une question d'honneur. Combattre un adversaire de beaucoup plus fort ne fait que les renforcer dans leur choix tragique. Le poème Tzar Lazar et tzarine Militza illustre ce sentiment du devoir à la fois religieux et national qui inspire les soldats serbes.

Le père de la tzarine Militza, le vieux Youg-Bogdan, avec ses neuf fils, ainsi que son mari tzar Lazar se préparent à partir pour le Kossovo ; la tzarine devrait rester seule à la maison. C'est pourquoi elle demande à son mari de laisser au moins un frère avec elle pour la protéger et servir de courrier entre elle et son mari. Le tzar consent et la tzarine part à la recherche de ses frères. Mais eux, l'un après l'autre, refusent la proposition et répondent :

«Va-t-en, ma sœur, va vers ta blanche tour,

Je ne voudrais, moi, guerrier, revenir en arrière

Ni abandonner les destriers du tzar,

Quand je saurais devoir périr.

Je vais, ma sœur, aux champs de Kossovo,

Verser mon sang pour notre Sainte Croix

Et pour la foi mourir avec mes frères. ${ }^{7}$ "

En les voyant tous partir, la tzarine Militza s'évanouit de douleur devant la maison. Son mari appelle alors le serviteur Goluban et lui ordonne :

"Goluban, mon serviteur fidèle,

Descends de ton cheval de cygne,

Prends ta maîtresse sur tes bras blancs,

Et porte-la jusqu'à la tour élancée.

Je te pardonne, au nom de Dieu,

Si tu ne vas pas te battre à Kossovo,

Mais reste ici dans mon blanc palais. ${ }^{8}$ "

Tout en pleurant, le serviteur Goluban obéit à son maître, il prend la tzarine Militza et la porte dans sa maison, «Mais à son cour il ne peut résister /et Kossovo l'appelle à la bataille. /Il revient vers son cheval de cygne, /le monte et s'élance vers Kossovo ${ }^{9}$ ".

Cet épisode montre à quel point le désir de combattre des soldats serbes a été irrésistible. Fidèles à eux-mêmes, à leur tzar, à leur empire, à leur religion, ils se précipitent tous à la mort. D'après les sources historiques, la bataille de Kossovo fut 
particulièrement sanglante pour les deux armées. ${ }^{10}$ Certains poèmes comme Le Serviteur Milutin illustrent l'ampleur de la catastrophe militaire serbe aussi bien que turque.

Le chant commence par l'arrivée de deux corbeaux dans la cour de la tzarine Militza, qui comprend vite que ces oiseaux noirs viennent du champ de bataille. Elle les supplie de raconter ce qu'ils ont vu sur la plaine de Kossovo, et les corbeaux répondent :

«Au nom de Dieu, tzarine Militza,

Nous venons ce matin des champs de Kossovo,

Et nous avons vu les deux armées terribles.

Les deux armées hier se sont heurtées

Et les deux tzars ont perdu la vie.

Il n'est rien resté des Turcs.

Mais il reste quelque chose des Serbes,

De la désolation et du sang ${ }^{11}$ ”

$\mathrm{Au}$ même moment arrive le serviteur Milutin portant «dans sa main gauche sa droite coupée; /son corps était criblé de dix-sept blessures /et son cheval ruisselait de sang ${ }^{12}$ ". L'image de ce serviteur estropié lors de la bataille démontre toute la férocité des combats. Ses forces revenues, Milutin raconte à la tzarine Militza le triste sort de son mari, de ses neuf frères, de son vieux père et celui de toute l'armée serbe :

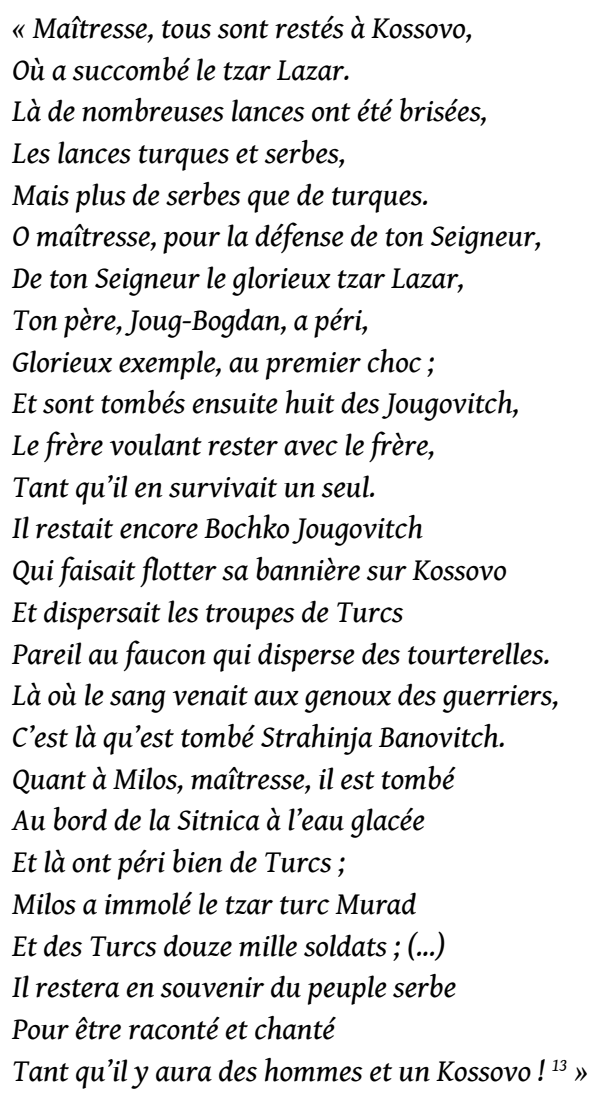

L'image de l'ennemi turc dans ce cycle de poèmes se reflète indirectement par la description du désastre de l'armée serbe. Illustrant la force des accrochages et le désarroi d'une armée vaincue, le poète confirme la puissance et la maîtrise extraordinaires des soldats turcs. L'arrivée inattendue de la peuplade asiatique est vécue et traduite dans l'esprit populaire serbe comme l'avènement d'un énorme monstre mythique, celui qui menace la vie et sème la mort. Devant une telle force étrange, on se sent paralysé, incapable de se défendre. En utilisant le langage mythique, 
le poète traduit la défaite militaire comme un coup du destin tragique. Ainsi, le chanteur épique crée un poème où la mère de Dieu, juste avant le combat à Kossovo, adresse une lettre au tzar Lazar en lui demandant de choisir entre le royaume céleste et le royaume terrestre :

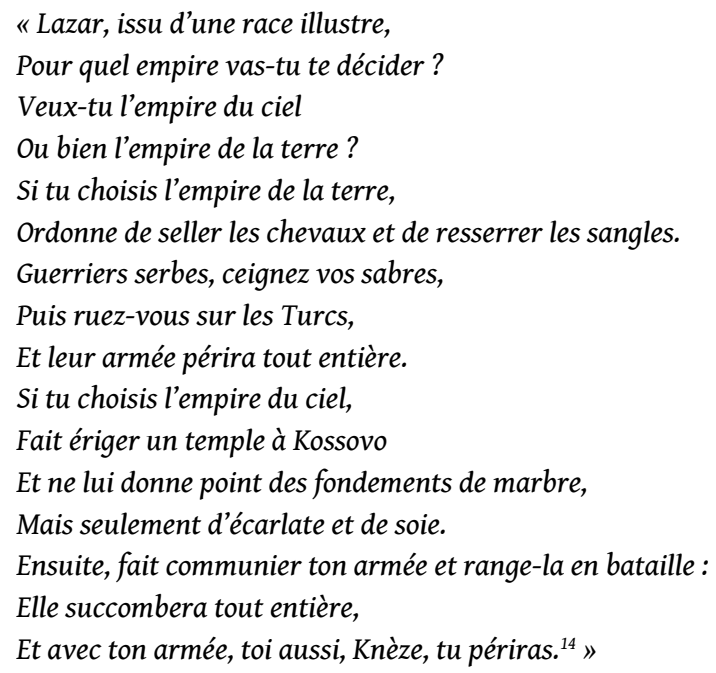

Le tzar Lazar a eu beaucoup de difficultés à faire le choix entre ces deux propositions. Finalement, il se décide et choisit l'empire céleste, car «l'empire de ce monde est bien passager et court, /tandis que l'empire du ciel durera dans les siècles des siècles ${ }^{15}$ ». Ainsi est justifié le mauvais sort du royaume serbe dans l'imaginaire populaire.

Mais dans le sacrifice du prince Lazar, notamment dans la scène de son départ avec son armée pour l'empire éternel, nous avons également les éléments d'une ancienne croyance provenant de la mythologie slave.

Dans l'épisode où la mère de Dieu envoie la lettre au tsar Lazar, on apprend que son message fut porté par Saint-Élie ${ }^{16}$. Cependant, les études ethnologiques montrent que, dans la tradition serbe, Saint-Élie incarne et hérite de l'image du dieu slave païen Perun, le dieu du tonnerre ${ }^{17}$. Selon cette ancienne croyance, le dieu du tonnerre est considéré comme le protecteur des guerriers qui après leur mort partent au ciel pour rejoindre Perun, leur maitre céleste. L'épisode du départ de l'armée serbe au ciel rappelle aussi des départs analogues présents dans d'autres systèmes mythologiques indo-européens. Dans ce sens, il est possible de constater certaines ressemblances entre l'envol de Lazar et de son armée et le départ des guerriers pour l'empire céleste décrit dans les mythologies germanique et scandinave. Le dieu suprême Odhinn recevait également les âmes des guerriers succombés dans les combats terrestres afin de composer son armée surnaturelle et exercer dans sa cour divine les batailles cosmiques éternelles.

Cette ancienne croyance slave marque profondément l'imaginaire collectif des Serbes dans lequel l'armée de Lazar est vécue comme l'armée sacrée et éternellement engagée pour défendre le peuple, son État et sa liberté. Les résurrections du peuple serbe pendant l'occupation turque ainsi que les différentes guerres de libération menées par l'armée serbe sont traduites dans l'esprit populaire comme l'aide portée au tsar Lazar et à son armée afin de vaincre l'ennemi et instaurer le royaume à l'instar de celui existant à l'époque du tzar ${ }^{18}$. 


\section{Le cycle de Marko Kraljevitch}

La bataille sanglante de Kossovo assena un coup mortel à l'empire serbe qui allait progressivement perdre son indépendance pour devenir en 1459 un territoire entièrement dominé par les Turcs. L'époque de la vassalité serbe est caractérisée par un regard plus nuancé sur les envahisseurs. L'image des Turcs reste imprégnée du sentiment de haine et de méfiance, toutefois elle se différencie de la présentation simpliste et répandue de l'ennemi féroce, exprimée dans le cycle de Kossovo. Cette première impression sur les Turcs dans laquelle ils rappellent une foule monstrueuse d'êtres surnaturels qui envahissent le royaume serbe évolue en devenant plus complexe et plus réaliste. Les Turcs sont maintenant présentés comme des hommes : il est vrai, sur le plan religieux, culturel ou celui de civilisation, ils se différencient de la population chrétienne autochtone, mais étant forcés de cohabiter avec les Turcs, les Serbes commencent à voir dans leurs adversaires de simples êtres humains.

Le cycle de poèmes consacré au héros national Marko Kraljevitch (le prince Marko) date de la période de vassalité où les Serbes vaincus apprennent à vivre sous la domination étrangère. De même que les personnages du cycle dédié à la bataille de Kossovo, celui du prince Marko est également une figure historique. Il était l'un des fils du roi Vukachine et il gouvernait une des plus grandes provinces serbes au XIV ${ }^{\mathrm{e}}$ siècle (la Macédoine). Afin de garder son identité culturelle et religieuse ainsi que ses biens, Kraljevitch Marko se fait vassal du sultan. Ce geste indigne d'un patriote n'a pas diminué sa popularité chez les Serbes. Dans l'imaginaire collectif, il est représenté comme une espèce de géant, d'une force physique prodigieuse et d'une humeur joyeuse et pacifique quand on ne lui cherche pas querelle; il devient dangereux quand il se sent provoqué, quand il se fâche. Selon la légende, Marko, accompagné de son fidèle cheval Šarac (Charatz), vécut trois cents ans et défendit le droit de ses compatriotes, soumis au pouvoir turc, le droit à l'identité nationale et culturelle.

Une dizaine de poèmes qui racontent les aventures du prince Marko reflètent également le changement d'attitude des Serbes envers les occupants. Leur héros, Marko, est si courageux et si glorieux, qu'il ne peut que servir l'empire, son égal en force et en importance. C'est à partir de cette idée que la vision des Turcs et de leur royaume change de caractère. Tout d'abord, l'occupation turque devient une réalité extérieure, un encadrement imposé dans lequel les Serbes sont obligés de vivre ; d'un autre côté, grâce à leur héroïsme et à leur adresse de combattants courageux, les Serbes jouissent en retour du respect des autorités turques, ce qui leur donne le sentiment d'être acceptés en égaux. Le héros Marko Kraljevitch exprime notamment tous ces défis et expériences.

Dans le poème Marko et Musa, nous avons l'illustration des relations complexes et ambiguës entre les Serbes et les Turcs. Oublié par le sultan, Marko passe sa troisième année enfermé dans la prison; soudainement, l'un des conseillers du chef turc se souvient du héros serbe et suggère au sultan d'aller le chercher, persuadé que seul Marko pourrait rendre service à l'Empire et résoudre le problème qui le perturbe : la révolte de Musa, célèbre guerrier turc devenu bandit.

Le poème se structure sur deux plans différents : en tant que vassal sans droit et ennemi des Turcs, le glorieux héros serbe est emprisonné, il est cependant libéré et accepté comme un combattant sans rival au moment où son adresse et son courage sont indispensables pour les intérêts de l'Empire. Puisqu'il n'y a que «Marko le fils de roi 
qui puisse nous délivrer de Musa ${ }^{19}$ ", le rebelle et le voleur dangereux qui extermine tous les braves soldats envoyés par le sultan, l'empereur fautif, tout en avouant que probablement «ses os sont déjà en poussière " car « il y a trois ans que j'ai envoyé Marko en prison et la prison n'a pas été ouverte depuis ${ }^{20}$ ", décide de le libérer de son cachot. Il promet à Marko de devenir le gouverneur de la Bosnie "pour neuf ans, neuf ans de suite » s'il accepte d'aller combattre Musa. C'est le moment où le rapport de forces change : Marko impose maintenant ses conditions et le sultan dépend de sa volonté.

"Seigneur tsar, par le Dieu puissant,

Le moisi de la prison m'a fait grand mal,

Je ne puis plus regarder de mes yeux:

Comment pourrais-je tenir au combat?

Fais-moi mener dans une hôtellerie,

Fais-moi donner force vin, force eau-de-vie,

Force chair de gras mouton,

Et avec cela du bon blanc pain,

Pour me refaire durant quelques jours ;

Je te dirai quand je serai en état de combattre ${ }^{21}$ "

Le sultan a rempli toutes les conditions du prince Marko qui, après quelques mois de repos demande qu'on lui apporte du bois sec de cornouiller afin de vérifier le niveau de sa force : «Il le presse fort dans sa main droite; le bois crève en deux ou trois endroits, et il en sort deux gouttes d'eau. Marko dit alors au glorieux tsar: maintenant, ô tsar, je puis combattre ${ }^{22}$ " Marko part chercher le bandit Musa célèbre pour son courage et son adresse de combattant. Ils s'affrontent sur le champ de bataille et finalement, c'est le prince serbe qui emporte la victoire. Musa tombe mort sur Marko qui voit trois cœurs dans la poitrine du bandit :

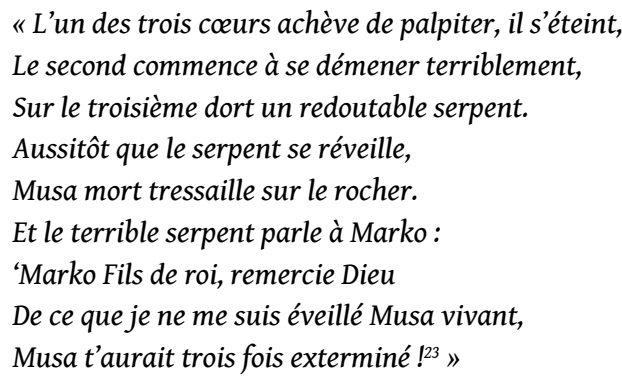

Marko réalise alors qu'il a tué meilleur héros que lui-même : "Malheur à moi, par le Dieu du ciel! /J'ai vaincu un plus brave que moi. ${ }^{24}$ ). Admettre que chez les Turcs, il peut y avoir des héros encore plus courageux que Marko, le plus fort de tous les Serbes, représente une nouvelle tendance dans la présentation des envahisseurs. Dans le cadre des valeurs propres à la morale héroïque, on accepte que les Turcs possèdent certaines qualités qui demandent le respect. C'est la raison pour laquelle, Marko Kraljevitch craint la vengeance des forces maléfiques qui peuvent punir le guerrier qui n'a pas honoré plus brave que lui. Superstitieux, Marko tremble de peur, car, en tuant le courageux Musa, il a perturbé l'ordre des valeurs héroïques, si important pour la société traditionnelle.

Bien que le prince serbe soit le vassal du sultan et qu'il participe souvent aux opérations militaires ottomanes, il garde ses origines chrétiennes et son caractère rebelle. Dans le poème Marko boit le vin pendant le ramadan, le célèbre héros provoque les autorités turques en refusant de respecter des lois proclamées par le sultan. Non seulement il s'habille en vert (la couleur verte était interdite aux chrétiens), mange et boit dans les auberges aux heures interdites, mais il invite également les musulmans et les représentants du sultan à partager le plaisir avec lui. Le tzar turc l'appelle et 
demande des explications. Lors de ce rendez-vous, en tête à tête avec le sultan, Marko montre à la fois une agressivité envers le chef turc et une prétendue naïveté grâce à laquelle il explique son geste de rébellion : il lui était impossible de manger et de boire seul, sans partager avec les autres. Le sultan ayant peur du prince serbe qui se montre aussi dangereux que fou, lui accorde le droit de faire tout ce qui était interdit auparavant et en plus "en cherchant dans ses poches, /le tzar sort cent pièces d'or, /il les donne toutes à Kraljevitch Marko:/va Marko, dit-il et boit le vin! ${ }^{25}$ "

Un autre poème devenu célèbre raconte les caprices du prince Marko. Il s'agit du chant intitulé Marko laboureur où le héros, à la demande de sa mère de renoncer à la vie de guerrier, prend la charrue et les bœufs et s'en va labourer la terre. La vie paisible des paysans n'est pas du goût de ce rebelle et il "prend la charrue et les bœufs, mais il ne laboure pas les monts et les vallées, il laboure les routes impériale ${ }^{26} »$. Pendant que Marko travaille les chaussées, les soldats turcs portant trois sacs d'or arrivent et exigent de lui d'arrêter et de les laisser passer : «Eh! Marko ne laboure pas les routes!». Le prince se fâche et leur rétorque : «Eh! les Turcs, ne piétinez pas les labours! ${ }^{27}$ »Les Turcs payeront cher leur refus de céder au prince serbe : énervé, Marko jette sa charrue et les bœufs, tue les soldats désobéissants et apporte à sa vieille mère les sacs d'or en lui disant: "Voilà ce que je t'ai ce matin creusé! ${ }^{28}$ ".

Considérant que le cycle des poèmes sur le prince Marko couvre l'époque de paix, c'està-dire l'époque où il n'y avait pas de guerre ouverte entre les Turcs et les Serbes, on peut constater que Marko, par son caractère rusé et sa maturité, rappelle le héros grec Ulysse. Tous deux sont à la fois de grands maitres d'armes, mais ce qui les distingue des courageux guerriers qui combattaient les Turcs à Kossovo ou les Troyens, c'est notamment leur envie de remporter une victoire sur la vie qui, maintes fois, est si dure et hostile à l'homme. Comme Ulysse, le prince Marko veut défendre son identité, ses origines, non seulement par la force de son poignet, mais également par celle de l'intelligence et la ruse. Puisque les deux symbolisent les héros civilisateurs qui montrent le chemin de la survie, ils possèdent aussi dans leurs caractères les éléments d'un trickster mythique ${ }^{29}$. Le prince serbe et le héros grec utilisent la tricherie, l'arme efficace pour lutter contre l'ennemi ou les difficultés imposées par le destin.

\section{Les chants héroïques sur les haïdouks et libérateurs serbes}

Ces traits de rebelle présents dans la personnalité de Marko Kraljevitch ne sont qu'une face de son caractère complexe. En jouant le trickster, le bouffon, il se permet de désobéir aux ordres turcs ; cependant, il n'entre pas ouvertement en conflit avec les occupants. Il sait qu'ils sont plus forts et pour lutter contre leur pouvoir absolu, Marko emploie aussi bien sa force physique que son esprit. Contrairement à lui, les héros de l'époque des haïdouks et plus tard de la période des guerres de libération ne font aucun compromis avec les autorités ottomanes. Le poète les présente comme les combattants libérateurs qui attaquent les Turcs par tous les moyens et pour un seul but : libérer le pays.

C'est notamment dans ces deux cycles de chants héroïques que l'image des Turcs revient à ses origines, celle du cycle sur la bataille de Kossovo. Cette fois, cependant, l'imaginaire populaire les voit comme des ennemis réels et féroces. Les sentiments de 
refus et de haine envers les Turcs, sentiments que le prince Marko cache derrière ses bouffonneries sont maintenant ouvertement exprimés.

Les chants consacrés aux haïdouks, et aux libérateurs de la Serbie dévoilent l'existence d'une méfiance réciproque qui, avec le temps, s'approfondissait dans les deux camps. La pression et l'hostilité des Turcs envers la population chrétienne ne s'arrêtaient pas au niveau des taxes et des dettes obligatoires que le raïa serbe devait payer. Afin de confirmer leur pouvoir absolu et stopper la révolte qui ne cessait pas d'augmenter au sein $\mathrm{du}$ peuple, les autorités ottomanes recourent à la force délibérée: elles pourchassent les familles entières, exécutent les hommes, violent les femmes ce qui ne faisait qu'aggraver une situation déjà dure et conflictuelle.

Dans le poème Doïtchin le Voïvode souffrant, nous avons le héros serbe qui, malade, ne pouvant plus assurer la protection de sa famille - sa femme et sa sœur - ainsi que de ses biens, fait l'objet d'une attaque sans pitié du Maure Houço qui « voyant qu'il n'y avait plus à Salonique de champions pour le combattre, /imposa une contribution à la ville: chaque maison devait lui envoyer un mouton, /une fournée de pain blanc, une charge du vin rouge,/une coupe d'eau-de-vie, ainsi que vingt jaunes pièces d'or, /et une belle fille, vierge ou nouvelle mariée ${ }^{30} »$. Lorsque ce fut au tour de la maison de Doïtchin de payer le tribut, la femme et la sœur du voïvode ne cachaient ni leurs larmes, ni leur tristesse. Sans protection, elles se préparaient au pire. Abandonné aussi par ses amis, le guerrier malade, Doïtchin, ordonne aux deux femmes de le préparer pour le combat: "Yelitza, ma chère sœur, apporte une pièce de toile, /afin que tu me serres les cuisses jusqu'aux côtes, /de crainte que mes os se déplacent et glissent les uns sur les autres ${ }^{31}$ ». Le voïvode, même souffrant, réussit à combattre le Maure et libérer de l'humiliation et de la peur non seulement sa maison, mais la ville entière. Il rentre chez lui et jette à sa sœur Yelitza les yeux du Maure : "ainsi tu sauras que tu n'as pas à les baiser, ma sceur, moi vivant. (...) /Et ayant ainsi parlé, le voïvode Doitchin rendit l'âme ${ }^{32}$ ».

La violence et l'humiliation auxquelles les Turcs ont recouru lors de leur occupation sont à l'origine de la révolte serbe. À leur fermeté, le raïa serbe répondait en formant les haïdouks qui vivaient dans les forêts et semaient la terreur et la peur parmi les envahisseurs. Le cycle des poèmes consacré aux haïdouks représente ainsi une sorte de témoignage historique. Il illustre non seulement la gravité du conflit entre les deux peuples porteurs de différentes religions et cultures, mais également la sévérité des beys turcs et la torture exercée dans leurs prisons.

L'exemple du poème Le petit Radojitza met en évidence le changement du rapport de forces qui s'annonce entre les Serbes et les représentants ottomans. Le chant commence par la fête que Bekir-bey organise au nom de sa grande victoire : il a réussi à capturer le plus grand haïdouk de sa région, le petit Radojitza.

"Non! ce n'est pas la foudre qui gronde,

Ce n'est pas la terre qui tremble,

La mer ne vient pas se briser sur les écueils

Et les Fées (Vilas) ne se battent pas dans la montagne.

Mais ce sont les canons qui grondent à Zadar,

Où le Bey Bekir-Bey se trouve en réjouissance de la prise du petit Radoïtza. $»^{33}$

L'ampleur de la fête du Bey démontre la profondeur de sa peur : c'est la joie de celui qui a survécu à sa mort. Après l'avoir battu, il met le haïdouk Radojitza en prison où se trouvaient déjà ses vingt camarades. Mais les rebelles serbes ne se découragent pas; le matin, ils appellent Bekir-Bey et lui annoncent que leur chef Radojitza est mort. Le Bey 
ordonne qu'on enterre le brigand, mais sa femme, se méfiant toujours de Radojitza, demande à son mari de vérifier s'il est vraiment décédé :

«Par Dieu! Radoïtza n'est pas mort

Et fait seulement de l'être.

Allumez-lui du feu sur la poitrine,

Nous verrons bien s'il bougera, le brigand. ${ }^{34}$ "

Comme Radojitza ne "fit pas le moindre mouvement", la femme du Bey continue sa torture. Elle demande qu'on apporte un serpent et qu'on le mette dans la chemise du haïdouk. «Mais le haïdouk avait un cour héroïque : / il ne bougea ni ne trembla. / Il ignorait la peur. » Toujours sûre d'elle-même, la femme du Bey poursuit ses vérifications : «prenez vingt clous et enfoncez-les lui sous les ongles. / Peut-être qu'alors il remuera, le brigand». À nouveau, Radojitza "montra la fermeté de son cœur. / Il n'eut pas un mouvement, n'exhala pas un soupir ${ }^{35}$ ". La femme du Bey ne renonce pas à ses investigations. Elle demande que les filles, avec la belle Haïkouna en tête, viennent et dansent devant Radojitza afin de voir s'il cèdera à la beauté. Et c'est son œil gauche qui trahit Radojitza et fait qu'il sourit à la belle Haïkouna. Ayant remarqué son sourire, la fille jette son mouchoir de soie sur le visage du haïdouk et dit à son père :

"Mon pauvre père, ne souille point ton âme d'un crime:

Fait emporter le captif et fait l'enterrer. ${ }^{36}$ "

À la demande de la femme du Bey, on a jeté Radojitza dans la mer pour que sa «belle chair [serve] de nourriture aux poissons ». Une fois dans l'eau, le haïdouk se sauve et prépare sa vengeance. Dès l'arrivée de la nuit, "il prit le chemin de la maison de Bekir-Bey » où le bey et sa femme se mettaient à dîner :

"Ma douce, ma fidèle épouse, voilà neuf ans que Rade s'est fait haïdouk

Et neuf ans que je ne pouvais souper tranquille, par crainte du petit Radoïtza.

Grâce à Dieu,j'ai pu m'en défaire : il n'est plus.

Demain, dès que le jour luira, je ferai pendre ces vingt autres. ${ }^{37}$ "

On devine facilement la fin du poème : Radojitza surprend le couple, décapite le Bey et réserve à sa femme une terrible vengeance. Il retire de sa poche les clous et les enfonce sous les ongles de la femme turque: "Mais il en avait à peine enfoncé la moitié, que la chienne expirait ${ }^{38}$ ». Ensuite, il cherche la fille du Bey, la belle Haïkouna qui lui ouvre le coffre aux pièces d'or et lui donne la clé de la prison. Après avoir partagé la richesse du Bey avec ses camarades, Radojitza prend Haïkouna et l'emmène chez lui : "Haikouna devint Angelia et Radoïtza la prit pour sa fidèle épouse ${ }^{39}$ \%.

La fin du poème montre que les rapports de forces entre le dominant et le dominé sont en train de changer. La belle fille du bey a pressenti que le haïdouk Radojitza, grâce à son endurance, emporterait la victoire dans le duel avec son père. Au moment où elle trahit ses parents et sauve le héros, elle tranche son propre destin. Jeune et belle, Haïkouna décide de suivre Radojitza. Son courage lui semble plus solide et moins étrange que les craintes et la férocité de ses riches parents.

À travers le destin des femmes, le chanteur épique annonce le changement politique. Ce ne sont plus les femmes serbes menacées par les Turcs (ce qui était le cas dans le poème de Doïtchin, le voïvode malade) ; l'épisode avec Haïkouna qui, convertie au christianisme, change de camp et devient Angelia et l'épouse fidèle de Radojitza, prédit symboliquement la victoire du raïa serbe sur les occupants turcs. Les Turcs avaient islamisé pendant leur règne une partie de la population chrétienne sont, à leur tour, exposés à la christianisation. 
Les guerres de libération du XIXe siècle sont suivies également par les chants héroïques qui célèbrent le courage revenu du peuple serbe. Le moment de revanche historique est venu et la défaite de Kossovo d'autrefois peut se transformer en victoire :

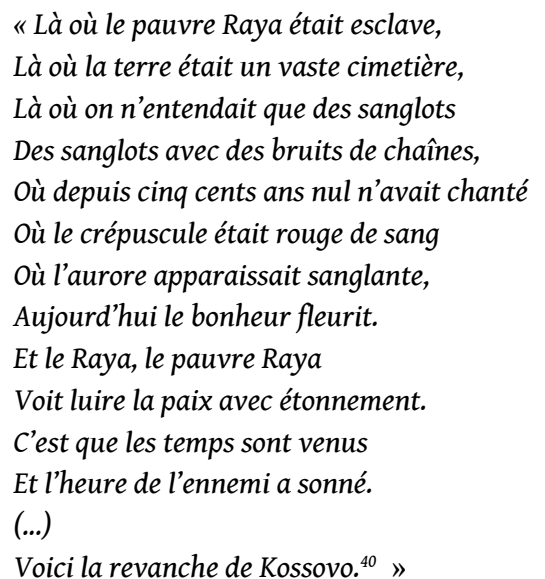

Pour les guerriers libérateurs du XIXe siècle, la bataille médiévale de Kossovo devient le symbole d'une bataille mythique. Pourchasser les Turcs cinq siècles après, cela signifie aussi réparer à la fois la défaite militaire et la gloire tragique des ancêtres. Le cycle des chants épiques sur Kossovo représente déjà une sorte de mythification des données historiques. Devenus les références emblématiques, quelques siècles plus tard, ces poèmes servent de modèle aux guerriers qui tentent de libérer le pays. Le passé déjà mythifié à travers l'imaginaire collectif se redresse et remythifie : le Kossovo devient le symbole de résistance et de courage, modèle qui instruit et nourrit la volonté et le désir de la liberté.

L'un des poèmes les plus connus qui racontent le soulèvement du peuple serbe, le soulèvement organisé et mené par Karageorges, le chef des insurgés, en 1804, est certainement celui créé par le rhapsode aveugle Filip Vichnjitch et intitulé : Le début de la révolte contre les potentats turcs. Le chanteur épique évoque un événement historique connu sous le nom du «carnage des princes serbes " par les Turcs. Ce massacre sauvage est finalement à l'origine de la révolte du peuple qui ne pouvant plus supporter l'injustice et la tyrannie des autorités ottomanes se soulève et se libère de l'esclavage.

Le chant commence par le rassemblement des Turcs qui, ayant peur du raïa serbe mécontent, décident de supprimer les représentants serbes les plus riches et les plus respectés afin de pouvoir mieux contrôler le reste du pays. Parmi les beys, le seul qui s'opposait à ce plan, c'était le vieillard Fotcho qui, dans leur geste de répression, reconnaissait la fin du régime ottoman. Le vieux Fotcho pense qu'il faut suivre l'exemple des sultans modérés d'autrefois qui n'étaient pas aussi durs avec leurs sujets. Convaincu que les conditions de vie aggravées, l'injustice et la force peuvent provoquer la révolte au sein du peuple, il conseille les jeunes beys de changer de stratégie. Car, "les routes désireront les Turcs, mais nulle part il n'y aura de Turcs ${ }^{41}$ ». Les beys, ne prenant pas en compte l'avis du vieillard, suppriment par surprise des centaines de chefs serbes et déclenchent ainsi la révolte du peuple. Menés par leur chef Karageorges, les rebelles s'organisent et débutent la vraie guerre de libération contre les occupants. Les combattants serbes retrouvent le moral de leurs ancêtres, et emportés par leur enthousiasme héroïque, ils réussissent à pourchasser les Turcs et à instaurer un nouvel État sous le règne de Karageorges. 
"Quand Karageorges sabre les Turcs,

Quand il sabre les Turcs oppresseurs,

Alors, seulement il entre dans les villes:

Que faire des Turcs dans les villes?

Les Turcs qui sont à sabrer, il les sabre,

Ceux qui sont à échanger, il les livre,

Il les a tous rendus (repris);

Ceux qui sont à convertir, il les convertit. ${ }^{42}$ "

Le poème de Filip Vichnjitch décrit non seulement le déroulement de la révolte du peuple serbe, mais également la création de son État moderne. De même que le prince Marko à l'époque médiévale, le chef des insurgés, Karageorges, devient le héros national, héros emblématique de la période des guerres de libération. Il est le libérateur et le fondateur de l'ère nouvelle dans l'histoire serbe.

\section{Conclusion}

Dans la présence turque, les Serbes ne voyaient pas seulement l'envahisseur cruel qui " brûlait les maisons, massacrait les enfants et violait les femmes ${ }^{43}$ ", mais surtout leur propre échec qui les fait appeler constamment à la vengeance et à la révolte. En ce sens, la poésie épique serbe de l'époque de la domination turque n'est qu'une longue épopée sur un thème unique : la liberté.

Ce qui leur était défavorable sur le plan historique - la perte de l'Empire serbe - les confortait, d'une certaine manière, sur le plan culturel. En perdant leur royaume terrestre, les Serbes gagnaient l'empire de l'esprit. C'est là où leur imaginaire collectif continue à créer leur portrait historique. La soif de liberté, l'envie de rester fidèle à leurs origines religieuses et culturelles, l'envie de préserver leur identité au prix d'une grande souffrance morale et matérielle ont pu être exprimées à travers la voix basse du chanteur épique. Grâce à leur tradition épique orale, les Serbes préservent leur langue, leur identité, leur histoire. La résistance culturelle s'avère ainsi comme la plus grande victoire.

\section{BIBLIOGRAPHIE}

Bakhtine, Mikhaïl, (1973), «Épopée et roman », dans Recherches internationales à la lumière du marxisme, $\mathrm{n}^{\circ} 76$, 3e trimestre, pp. 5-39.

Bakić-Hayden, Milica, (2006), Varijacije na temu Balkan [Variations sur le thème des Balkans], Belgrade : Filip Višnjić, 161 p.

Bataković, Theodor Dusan, (dir.), (2005), L'Histoire du peuple serbe, Lausanne : L'Âge d'Homme, $279 \mathrm{p}$.

Bojović, Bosk, (1995), L'idéologie monarchique dans les hagio-biographies dynastiques du Moyen Âge serbe, Roma : Pontifico Istituto orientale, $727 \mathrm{p}$. 
Braun, Maximilien, (2004), Srpskohrvatska junačka pesma [Le poème hérö̈que serbo-croate], Belgrade : Matica Srpska et Vukova Zadužbina, $278 \mathrm{p}$.

Čajkanović, Veselin, (1973), « O Vrhovnom bogu u staroj srpskoj religiji », [Le dieu suprême dans l'ancienne religion serbe], in Mit i religija u Srba, [Mythe et Religion chez les Serbes], Belgrade : SKZ, pp. 307-463.

Calvet, Louis-Jean, (1984), La tradition orale, Paris : PUF, 126 p., $2^{\mathrm{e}}$ éd.

Djurić, Vojisla, (1977), Antologija junačkih narodnih pesama [Anthologie des poèmes héroïques populaires], Belgrade : SKZ, $772 \mathrm{p}$.

Dumézil, Georges, (1959), Les dieux des Germains, Paris : PUF, 128 p.

Dvornik, Francis, (1970), Les Slaves, Paris : Seuil, 1178 p.

Gezeman, Georg, (2002), Studije o južnoslovenskoj narodnoj epici, [Études sur la poésie épique des Slaves du Sud], Belgrade : Matica Srpska et Vukova Zadužbina, 230 p.

Gruel-Apert, Lise, (1995), La tradition orale russe, Paris : PUF, 300 p.

Ibrovac, Miodra, (1966), Claude Fauriel et la fortune européenne des poésies populaires grecque et serbe, Paris : Didier, $719 \mathrm{p}$.

Jovanović, Vladislava, (1979), Srpske narodne pesme, [Les poèmes populaires serbes], Belgrade : Slovo ljubve, $340 \mathrm{p}$.

Loma, Alexandar, (2002), Prakosovo [Pra-Kossovo], Belgrade : SANU, 352 p.

Mélétinsky, Evgeni, (1996), « La poétique historique du folklore narratif », dans Ethnologie française, Tome XXIV, Paris : octobre-décembre, pp. 611-619.

Miletich, John, (1990), The Bugarstica, Chicago: University of Illinois Press, 339 p.

Orfer, de Léo, (1916), Chants de guerre de la Serbie, Paris : Payot, 254 p.

Soerensen, Asmus, (1999), Prilog istoriji razvoja srpskog junačkog pesništva, [Une contribution à l'évolution historique de la poésie épique serbe], Belgrade : Matica Srpska et Vukova Zadužbina, $373 \mathrm{p}$.

Vaillant, André, (1949), « Marko Kraljević et son frère Andrijaš », dans Annuaire de l'Institut de philologie et d'histoire orientales et slaves, Tome IX, Bruxelles, pp. 569-575.

Worthington Ian et Foley John Miles, (2000), Epea and Grammata, Boston Köln : Brill, pp. 3-25.

\section{NOTES}

1. M. Ibrovac, Claude Fauriel et la fortune européenne des poésies populaires grecque et serbe, Paris, Didier, 1966, p. 70 : «C'est lui (Kopitar) qui a guidé et soutenu, dans sa lutte héroïque contre des préjugés séculaires, l'autodidacte de génie que fut Vuk Karadjitch, le père de la littérature serbe moderne ; lui, qui, le premier, a fait, ou suscité, des traductions allemandes des chants serbes et grecs afin de les soumettre à Goethe, à Grimm, à Werner von Haxthausen, l'émule allemand de Claude Fauriel. »

2. Ibidem, pp. 409-601.

3. L. D’Orfer, Chants de guerre de la Serbie, Paris, Payot, 1916, p.41.

4. V. M. Jovanović, Antologija srpske narodne poezije [Anthologie de la poésie épique serbe], Beograd, Slovo ljubve, 1979, p.96.

5. L. D’Orfer, op. cit., p. 45. 
6. Ibidem, p. 53.

7. Ibid., pp. 54-55.

8. Ibid., p. 55.

9. Ibid., p. 56.

10. Dušan Bataković, (dir.), L'Histoire du peuple serbe, Lausanne, L'Âge d'Homme, 2005, p. 63 : "Dans une chronique parue au début du $\mathrm{XV}^{\mathrm{e}}$ siècle à Florence, on affirmait que le sultan Murat avait envoyé 140000 soldats en Serbie, alors que le prince Lazar lui avait opposé une force inférieure de moitié, et qu'il y avait eu 30000 Serbes tués ainsi que 70000 Turcs; parmi ces derniers, il y aurait eu 20000 tués par les habitants des pays traversés pendant leur retraite.»

11. L. D’Orfer, op. cit., p. 57-58.

12. Ibidem, p. 58.

13. Ibid., pp. 59-60.

14. Ibid., pp. $42-43$.

15. Ibid., p. 43.

16. Ibid, p. 42 : «Un faucon au plumage gris arrive à tire-d'aile /Des Lieux saints, de la cité de Jérusalem, /Et il porte une légère hirondelle. /Ce n'est pas un oiseau, ce n'est pas un faucon gris, /Mais bien le prophète Saint-Élie; »

17. Voir : Veselin Čajkanović, "O Vrhovnom bogu u staroj srpskoj religiji », [« Le dieu suprême dans l'ancienne religion serbe »], in Mit i religija u Srba, [Mythe et Religion chez les Serbes], Belgrade, SKZ, 1973, pp. 307-463. ; Aleksandar Loma, Prakosovo, [Pra-Kossovo], Belgrade, SANU, 2002, pp. 150-151.

18. M. Bakić-Hayden, Varijacije na temu Balkan, [Variations sur le thème des Balkans], Belgrade, Filip Višnjić, 2006, pp. 124-143.

19. M. Ibrovac, op. cit., p. 627.

20. Ibidem, p. 628.

21. Ibid., p. 628.

22. Ibid., p. 629.

23. Ibid., p. 632.

24. Ibid., p. 632

25. V. Djurić, Antologija narodnih junačkih pesama [Anthologie des poèmes héroïques populaires], Beograd, SKZ, 1977, p. 320.

26. Ibidem, p. 347.

27. Ibid.

28. Ibid.

29. Voir : S. Bošković, "L'Odyssée d'Homère et l'Ulysse de Joyce », dans La poétique du mythe dans la littérature contemporaine, Lille, Thèse, 2002. pp. 69-83.

30. L. D’Orfer, op. cit., p. 186.

31. Ibidem, pp. 189-190.

32. Ibid., p. 193.

33. Ibid., p. 161.

34. Ibid., pp. 162-163.

35. Ibid., p. 163.

36. Ibid., p. 163.

37. Ibid., p. 165.

38. Ibid., p. 166.

39. Ibid., p. 167.

40. Ibid., pp. 214-215.

41. V. Djurić, op. cit., p. 655.

42. Ibidem, p. 666.

43. Ibid., p. 660. 


\section{RÉSUMÉS}

L'œuvre de Vuk Karadjitch et son évocation de l'image du Turc dans la poésie épique serbe. Persuadé que la poésie épique serbe porte une valeur exceptionnelle dans la formation de la langue elle-même ainsi que dans la constitution de l'identité nationale et de la mémoire collective, Vuk Karadjitch se hâte, durant toute sa vie, de rencontrer les chanteurs épiques-aèdes pour les écouter et noter les poèmes. C'est ainsi qu'il réussit à recueillir environ mille chants populaires.

Les critères de systématisation de Vuk Karadjitch prennent en compte le contenu thématique des poèmes. Il divise les chants en deux familles : les poèmes féminins traitant des sujets appropriés, et les poèmes masculins qui évoquent les thèmes héroïques, les batailles, les guerres, les personnages et les événements importants de l'histoire. Les chants masculins sont au centre de nos recherches dans cette étude. Ces poèmes qui parlent des batailles sanglantes et de l'héroïsme national se situent historiquement à l'époque de l'envahissement turc des territoires serbes. Selon les différentes périodes de l'occupation ottomane, on peut voir l'évolution de l'image de l'occupant turc, construite dans l'imaginaire collectif.

Dans la présence turque, les Serbes ne voyaient pas seulement l'envahisseur cruel, mais surtout leur propre échec qui les fait appeler constamment à la vengeance et à la révolte. En ce sens, la poésie épique serbe de l'époque de la domination turque n'est qu'une longue épopée sur un thème unique : la liberté.

Persuaded that epic Serbian poetry plays an exceptional part in the formation of the language itself as well as in the constitution of a national identity and collective memory, Vuk Karadjich hurries throughout his life to meet traditional epic-singers and note down their poems. It is in this way he is able to collect around one thousand popular songs.

Vuk Karadjich's criteria for systematisation take into account the thematic content of the poems. In his collection, he divides the songs into two categories: the female poems, dealing with appropriate subjects, and the masculine poems, evoking heroic themes, battles, wars, important people and events of history. The masculine songs are also at the centre of our research in this study. These poems of bloody battles and of national heroism are situated historically during the time of the Turkish invasions of the Serbian territories. Following the different periods of the Ottoman occupation, we can see the evolution of the image of the Turkish invader formed in the collective imaginary.

In the Turkish occupation, the Serbs do not only see the cruel invader but above all they see their own adversity which makes them constantly call for vengeance and revolt. In this sense, the epic Serbian poetry from the era of the Turkish domination is but one long epic on a single theme: liberty. 
INDEX

motsclestr Sırbistan, Kosova, Krousovo, Osmanlı İmparatorluğu

Thèmes : Littérature

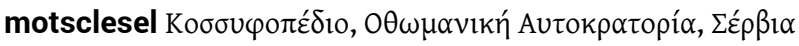

Index chronologique : Empire ottoman, dix-neuvième siècle

Mots-clés : Karadžić Vuk Stefanović (1787-1864), Haïdouk/Haïduk, Kraljevic Marko/Prince

Marko (1335-1395), poésie épique serbe, Ottomans dans la littérature

Index géographique : Kosovo, Serbie

motsclesmk КОСОВО, ОТОМАНСКАТА ИМПЕРИЈА, СРБИЈА

Keywords : Karadžić Vuk Stefanović (1787-1864), servian poetic poetry, Literature, Ottoman empire, Serbia, nineteenth century 